\title{
Prevalence of Irritable Bowel Syndrome among Nursing Students at King Abdulaziz University, Jeddah, Saudi Arabia
}

\author{
Ahlam Al.qarni, Mona Almutairi, Muna Bamatraf, Ruba Alharbi*, Samia Alsulami, Fatmah Alsharif \\ Nursing, King AbdulAziz University, Jeddah, Saudi Arabia \\ *Corresponding author: Rubaalharbi@outlook.com
}

Received May 09, 2019; Revised June 20, 2019; Accepted July 01, 2019

\begin{abstract}
Introduction: Irritable bowel syndrome (IBS) is a common functional gastrointestinal disorder that may be triggered by enteric pathogens. Research problem: Studies show IBS syndrome is a common disorder worldwide, for example 5-20\% in the United States have irritable bowel syndrome, mostly among adults with age $<50$ years old. Also, there is a study conducted in Al-Ahsa in 2017 that show the prevalence of IBS between medical student and interns in Saudi Arabia is 31.8\%. Another study was conducted in Hail in 2018 among medical students show that $18 \%$ of the participants have been diagnosed with irritable syndrome. Moreover, no study was conducted exclusively among females nursing students in Jeddah. Materials: The questionnaire was adapted from a validated survey that was previously used. Method: A cross-sectional descriptive study was conducted among 193 undergraduate nursing students (second, third and fourth years) at King Abdul-Aziz university. Results: The results showed that $17.6 \%$ of nursing students at King Abdul-Aziz having IBS. Conclusion: The results showed that the prevalence of IBS is $17.6 \%$. Recommendations: We recommend Screening for IBS and psychological problems and Stress management course. Also, we recommend to ministry of education to provide relaxation room for students at colleges.
\end{abstract}

Keywords: irritable bowel syndrome, IBS, nursing students, prevalence

Cite This Article: Ahlam Alqarni, Mona Almutairi, Muna Bamatraf, Ruba Alharbi, Samia Alsulami, and Fatmah Alsharif, "Prevalence of Irritable Bowel Syndrome among Nursing Students at King Abdulaziz University, Jeddah, Saudi Arabia." American Journal of Nursing Research, vol. 7, no. 5 (2019): 677-683. doi: 10.12691/ajnr-7-5-2.

\section{Introduction}

"Irritable bowel syndrome (IBS) is a common functional gastrointestinal disorder that may be triggered by enteric pathogens and has also been linked to alterations in the microbiota and the host immune response" [1].

Studies show IBS syndrome is a common disorder worldwide, for example 5-20\% in the United States have irritable bowel syndrome, mostly among adults with age $<50$ years old [2]. In addition, the prevalence of irritable bowel syndrome in Saudi Arabia is 31.8\% among medical students and interns in Saudi Arabia [2].

There are fourth classifications of pathophysiology of irritable bowel syndrome. First, the changes in gastrointestinal motility, it considers disorder of irritable bowel syndrome patient with motor abnormalities in the colon and small bowel. Second, the abnormal gas handling the major complaint of patients irritable bowel syndrome bloating and abdominal distance. In normal processing of gas inside small bowel, the gas not remain, but the patient irritable bowel syndrome experience amount of gas remains in the bowel. Third, psychosocial factor affective disorder, involving somatization, anxiety, hostility and phobia this major combined condition with irritable bowel syndrome. Finally, gestational infection. some factor improvement post infection irritable bowel syndrome involves virulence, age, and female gender [3].

The change in stool form and frequency is the best important step to make a positive diagnosis with irritable bowel syndrome, so these changes allowed to classify the irritable bowel syndrome to subgroups based on predominant stool pattern experience [4]. They are diarrhea - predominant (IBS-D), Constipation - predominant (IBS-C) or those who fluctuate or mixed between the two which called (IBS-M). (IBS-D): The intestines are very much irritated, and the bowel movements will be very fast. Therefore, they will be passing a lot of loose stools frequently. The person tends to experience diarrhea first things in the morning or after eating due to exaggerated gastrocolic reflex and almost never at night also it Increases the person's sense of incomplete emptying of the rectum. His loss or watery stools $\geq 25 \%$. They need to toilet is typically urgent and can't be delayed it. (IBS-C): This disturbed occurs due to low fiber diet in daily habits and straining for a long time, the person tends to alternate constipation with normal hard or lumpy stools $\leq 25 \%$. In this type, pain occurs in attacks, usually relieved by defecation and sometimes provoked by food.) IBS-M): it 
is called alternative type, which is a mixture of the constipation and diarrhea [5].

Irritable bowel syndrome results from a change in intestinal mortality, this is may be due to several reasons such as: Neuroendocrine dysreglration of some chemicals made by the body, such serotonin and gastric, Infection, or gastroenteritis such as SIBO - $\mathrm{H}$. pylori and overgrowing of yeast may involve changes in peristaltic movement, food sensitivities can also, irritate the bowel. In addition, hormonal fluctuation affects bowel function in women, especially in menses cycle that can increase rectal sensitivity [6].

Irritable bowel syndrome is associated with hereditary factors, psychological factors and lifestyle habits. Psychological factors such as anxiety, and depression [7]. Lifestyle habits such as follows diet high in fat or irritating food, and smoking [6]. In addition, there are emerging factors related to irritable bowel syndrome such as intestinal microbiota, intestinal permeability, and altered gut immune activation [8].

Regarding the diagnosis of irritable bowel syndrome, there is diverse diagnostic criteria have been set up, for example, Manning criteria, Rome I, Rome II, Rome III and Rome IV. The first one was called Manning criteria, published in 1978 [9].

According to the Rome Foundation criteria, we have four different versions Rome I, II, III and IV [9]. The Rome criteria were established to recognize functional gastrointestinal disorders (FGIDs), which are conditions that are based on symptoms that dissented to conditions that are defined by pathological organ [10].

Rome I, the first edition published in1991, is comparatively complex and there is difficult to use and were later modified [3]. Rome II, the second edition, is focus on the frequency of symptoms occurring twelve weeks (not necessarily successive weeks) within one year [11].

However, Rome III criteria it is the third edition published in 2006. It is used clinically for detection of IBS which defines as " abdominal pain or discomfort more than 3 days per month over the previous 3 months associated with disturbed defecation or a change in bowel habit associated with two or more of the following: Improvement with defecation, Onset associated with a change in frequency of stool and Onset associated with a change in shape of stool" [12].

The recent criteria is Rome IV, released in May 2016. There is a few changes between Rome III and Rome IV. One of these changes is the term of discomfort is eliminated. Therefore, only abdominal pain is included. Also, 'Improvement with’ defecation has been replaced by 'Related to' defecation, and 'Onset' has been excluded from the associated changes in frequency and change in shape of stools [13].

The major treatment of irritable bowel syndrome is (loperamide) effected change the bowel habits of irritable bowel syndrome patients. The other treatment (Tegaserod) most effective, reducing some symptoms in women with irritable bowel syndrome [14].

A vast majority of researches describe and focus on nurse role in different settings [15-22]. Research problem Studies show IBS syndrome is a common disorder worldwide, for example 5-20\% in the United States have irritable bowel syndrome, mostly among adults with age $<50$ years old. Also, there is a study conducted in Al-Ahsa in 2017 that show the prevalence of IBS between medical student and interns in Saudi Arabia is 31.8\% [2]. Eventually it would affect their academic performance and achievements. Moreover, no study was conducted exclusively among females nursing students in Jeddah.

\section{Aim of the Study}

The study was conduct to assess the prevalence of IBS among nursing students at king Abdul-Aziz University.

\section{Research Question}

What is the frequency of IBS among nursing students at king Abdul-Aziz University?

\section{Literature Review}

\subsection{Prevalence of IBS Types}

Several researches studied the prevalence of different types of irritable bowel syndrome such as a research that was conducted by Nahla Ibrahim and colleagues in (2016), which are a cross-sectional study to define the occurrence, Intensity and predictors of irritable bowel syndrome among nurses employed at King Abdul-Aziz University Hospital in Jeddah, Saudi Arabia [23]. They collect the data from 229 nurses working in KAUH using Rome III criteria to determine the propagation of IBS which was $14.4 \%$ and regarding the IBS subtypes prevalence was $54.5 \%$ for IBS-Mixed, $27.3 \%$ for IBS-C, $12.1 \%$ IBS-U and $6.1 \%$ for IBS-D [23]. In comparison to Yang Liu and colleagues in (2014), they determine the prevalence of IBS and it is related factors through self-administered questionnaires using Rome III criteria [24]. The study was conducted at Beijing University of Chinese Medicine, among 843 medical students [24]. The result show that 255 of them are IBS positive, and about the subtypes 112 of them are IBS-Mixed with a prevalence of $43.9 \%, 79$ are IBS-D (31.0\%), 49 are IBS-U (19.2\%), and 15 are IBS-C (5.9\%) [24]. Additional research was conducted by Saad Naeem and colleagues in (2012), that investigate the prevalence of irritable bowel syndrome and it is relationship with anxiety using Rome III Criteria and generalized anxiety questionnaire. All the student that came out irritable bowel syndrome negative were used as control and the 102 out of 360 who were positive used as cases [25]. About the subtypes 55\% of them are case of IBS-Mixed and 25\% of the 102 are a case of IBS-C [25]. Furthermore, IBS-M was found to be the most common type of IBS according to Nahla Ibrahim (2016), Yang Liu (2014) and saad Naeem (2012) [23,24,25].

\subsection{Factors Related to IBS}

A considerable amount of literature has been published on Irritable bowel syndrome. These studies showed the results of most common risk factors of IBS. In a study carried out by Al-Turki Yousef and colleges (2011), to 
assess the prevalence of Irritable Bowel Syndrome (IBS) between King Saud University (KSU) students, Riyadh, Saudi Arabia, by taking the lifestyle and stressor in consideration. [26]. It is a cross-sectional survey among KSU students [26]. The data was collected via selfadministered questionnaires. From 1350 students was (54.4\%) of them have a Psychological stress and (60.8\%) those who have insomnia with IBS [26]. In addition a cross-sectional design was accomplished to study the prevalence and risk factors of irritable bowel syndrome among medical students of Peshawar, Pakistan by Bushra Iftikhar and colleges (2018) [27].

It is using simple random sampling technique. Standardized measuring scales/questionnaires were used to determine IBS and its related factors. Which is found 156 students (28.3\%) from 552 having high degree of stress, 21.8\% of these cases were diagnosed with IBS. 336 students (60.9\%) were having moderate degree of stress, $10.7 \%$ of these cases were diagnosed with IBS. 60 students (10.9\%) having mild level of stress, $6.7 \%$ of these cases were diagnosed with IBS. The connection of IBS disorder was also found with sleep disturbances in the same students. 360 students (65.2\%) were having perfect quality of sleep, $10.6 \%$ of these cases were diagnosed with IB. 118 students (21.4\%) with acceptable degree of sleep, 20.3\% were diagnosed to have IBS. 74 students (13.4\%) with poor quality of sleep, and $16.2 \%$ of these cases were diagnosed with IBS [27]. Additional research conducted by Nahla Ibrahim and colleges (2013) on 597 medical and interns students at King Abdul-Aziz University. A cross-sectional study, Self-administered questionnaire was used. The results revealed that $40.1 \%$ of the cases were positive for IBS, experienced emotional stress in the 6-months and only $20.1 \%$ of them who did not have stress. Overall, high level of stress and insomnia was found to be strongly associated with irritable bowel syndrome according to previous studies [12].

In contrast to the previous studies, has been identified another risk factors such as a cross-sectional observational study that was conducted among 270 academic medical students at King Saud bin Abdul-Aziz University for Health Sciences (KSAU-HS) by Alaqeel and colleges (2017) to recognize the relationship between anxiety and IBS [2]. The result was 39\%, 7\%, and $25.9 \%, 13.3 \%$ and $14.1 \%$ of students had normal, mild, moderate, severe and highly severe levels of anxiety [2]. In addition, this is an observational and cross-sectional study conducted by Yukiko Okami and colleges (2011) to explore the relationships between IBS and lifestyle factors in 2,639 nursing and medical students [28]. The result was related to anxiety factor in different gender In males $\mathrm{p}=0.001$; in females, $\mathrm{P}=0.001$. On the other hand, depression factor play role on IBS increasing .regarding to males $p=0.037$; In Females $\mathrm{p}=0.001$. Eventually, There is a relatively relationship between depression and anxiety to IBS especially in medical and nursing students [28].

\section{Methodology}

\subsection{Design}

The research design is a descriptive cross-sectional design that was conducted at King Abdul-Aziz University, in the faculty of nursing from February 2019 to March 2019, which is a "primary none-experimental research design" [29]. The researchers were chosen this study design because it is the most appropriate design for our research study and this study design does not require a specific time to collect data or to follow up with the participants. In addition, this study design was used to reach large numbers of participant quickly via online survey.

\subsection{Study Setting}

This study was implemented in the faculty of nursing at King Abdul-Aziz University in Jeddah, Saudi Arabia. The faculty characterized by having 101 employees, 385 students, and 100 faculty staff with PHd degree, MSn and bachelor's degree in nursing. The reason behind selecting this organization is that the scarcity of research linking the relationship between female nursing students and irritable bowel syndrome and its associated factors in Saudi Arabia.

\subsection{Sampling and Sample Size}

The sample group include all undergraduate nursing students (second, third and fourth years) at King AbdulAziz University in the faculty of nursing. This sample was chosen because we are nursing students and we are more susceptible to irritable bowel syndrome. A sample size is a subset of the population (is a group selected from a population) [30]. A convenience technique used by distributing online survey. The researcher's inclusion criteria are female undergraduate nursing students (second, third, fourth years) at King Abdul-Aziz University. The researcher's exclusion criteria are the other medical students and non-medical students at King Abdul-Aziz University, nursing staff and nursing interns.

In this study proposed to determine the representative sample, the total numbers of the population of all undergraduate nursing students (second, third and fourth years) at King Abdul-Aziz University are 385. The confidence level is (95\%) and desired confidence interval is (5\%) using the following equation http://www.raosoft.com/samplesize.html. Based on the calculated equation above, the sample size of this proposed study was calculated as follows. Sample size for all undergraduate nursing students (second, third and fourth years) at King Abdul-Aziz University is 193 students.

\subsection{Ethical Considerations}

Ethical approval was obtained from the faculty of nursing at King Abdul-Aziz University in Jeddah. No harm will be imposed on the participants and their rights will be respected and protected. Researchers aim to fulfill the ethical duty of protecting participants' information and confidentiality. Furthermore, researchers are seeking to collect and access information about participants that completely protects their anonymity. Information or data that possess identifiable qualities can indisputably stir the participants' confidentiality.

In addition, researchers will protect participants' information on the private computer with a locked 
password and for access only for the researchers and the data will be destroyed after one year for publication reason.

\subsection{Instrumentation}

In this study, the researchers used an adapted questionnaire from Almezani and colleagues (2018). The survey was used in Saudi Arabia, among Medical Students in Hail University and it was prepared in Arabic language [31].

"The questionnaire includes 14 questions it consisted of two parts. Part one: demographic data that includes gender, age, marital status, educational level. part two: the students will be asked whether they have been currently diagnosed with irritable bowel syndrome or not, whether they have been experiencing abdominal pain, discomfort (one or more) in the last three months and whether it increase or decrease with defecating and if they have changes in their bowel habit or stool consistency if they have not been diagnosed with irritable bowel syndrome students were also asked if the current abdominal pain has affected their academic achievements at university or not, if there are suffering from anxiety or not and whether they are smokers or not. Also, if they have someone in their families who has been diagnosed with irritable bowel syndrome or not, in last if they exercise regularly or not" [31]. The participating response was measured by using yes or no answers.

\subsection{Data Collection Procedure}

The data were collected by using an online questionnaire which can cover a wide area, it considers the only method, the researcher can collect numerous and accurate data directly from participants from large population with reasonable economical way. As well, it is helpful for saving researchers time. In other word, We have been to use the online questionnaire because it is be responsible for privacy of the participants in the questionnaire, which is one of the most important rights, so it was easy to use this kind of online questionnaire. That sent to a voluntary participant in the study who agrees through an implied consent form. The process where they are answering the questions will take approximately five minutes.

\subsection{Data Analysis}

Data was analyzed by using descriptive statistics through SPSS version 23 in the form means, percentage, frequency and standard deviations.

\section{Results}

A total of one hundred and ninety-three nursing students sign the online consent form to participate in this study. Table 1 showed that 139 students with a percentage of $72.0 \%$ have age range between 19-21 years, and 54 students with a percentage of $28.0 \%$ have age range from 22-24. It also shows that a total of 189 (97.9\%) participants are single while only four participants $(2.1 \%)$ are married. Regarding their educational level, Table 1 demonstrated that the majority of the nursing students $(n=65 ; 33.7 \%)$ are in their second year, while a total of 64 (33.2\%) nursing students are in their third year and $n=64$ (33.2\%) in their fourth year. Table 2 revealed that the prevalence of IBS among nursing student at King Abdulaziz University is $17.6 \%$. And ninety-one students with a percentage of $47.2 \%$ reported that they experience recurrent abdominal pain (one or more) in the last 3 months while $44 \%$ did not experience any recurrent abdominal pain. And 58 students with a percentage of $30.1 \%$ report that the pain increases with defecating and 89 students with a percentage of $46.1 \%$ their pain did not increase with defecating. Going to question number eight in Table 2 that ask them "Did you notice any changes in your stool consistency, fourty eight students with a percentage of $(24.9 \%)$ noticed a change in their stool consistency. On the other hand, a total of 145 nursing students did not notice any changes in their stool consistency. Considering the bowel habits, 78 out of 193 (40.4\%) of nursing students noticed a change in their bowel habits as shown in Table 2 whereas a total of 115 did not noticed any changes. If we now turn to question number 10 the students were asked "Did the abdominal pain affect your academic performance” and it shows that 39 students with a percentage of $20.2 \%$ reported that abdominal pain affects their academic performance and surprisingly 154 students says it did not. The most surprising aspect of the data is in question number 11 it ask the students "Do you have anxiety?” and it reveals that one hundred and thirty-eight students with a percentage of $71.5 \%$ are experiencing anxiety and only 55 students are not. Turning now to the next question the students were asked "Are you a smoker?" and it shows that only fifteen students with a percentage of (7.8\%) are smokers and one hundred and seventy-eight (92.2\%) are non-smokers. Question number 13 of the survey which ask the students "Do you have family history of irritable bowel syndrome?" and it reveals that ninety-eight students with a percentage of (50.8\%) report that they have family history of IBS and ninety-five student report that they do not. In the last question of the survey, respondents were asked "Do you exercise regularly" and 27 students with a percentage of (14\%) said yes and surprisingly 166 students with a percentage of (86\%) said no.

Table 1.

\begin{tabular}{|c|c|c|}
\hline \multicolumn{3}{|c|}{ General Characteristics (Demographic) } \\
\hline Characteristic & Frequency & Percentage \\
\hline Female & 193 & 100 \\
\hline \multicolumn{3}{|c|}{ Age(years) } \\
\hline $19-21$ & 139 & 72 \\
\hline $22-24$ & 54 & 28 \\
\hline \multicolumn{3}{|c|}{ Marital status } \\
\hline Single & 189 & 97.9 \\
\hline Married & 4 & 2.1 \\
\hline \multicolumn{2}{|c|}{ Gender } & 33.7 \\
\hline Second year nursing & 64 & 33.2 \\
\hline Third year nursing & 64 & \\
\hline
\end{tabular}


Table 2.

\begin{tabular}{|c|l|c|c|}
\hline NO. & Questions & 1. yes (\%) & 2. no (\%) \\
\hline 5. & Have you been diagnosed with irritable bowel syndrome? & $34(17.6)$ & $159(82.4)$ \\
\hline 6. & Did you experience recurrent abdominal pain (one or more) in the last 3 months? & $91(47.2)$ & $85(44.0)$ \\
\hline 7. & Dose the pain increase or decrease with defecating? & $58(30.1)$ & $89(46.1)$ \\
\hline 8. & Did you notice any changes in your stool consistency? & $48(24.9)$ & $145(75.1)$ \\
\hline 9. & Have you noticed any changes in your bowel habits? & $78(40.4)$ & $115(59.6)$ \\
\hline 10. & Did the abdominal pain affect your academic performance? & $39(20.2)$ & $154(79.8)$ \\
\hline 11. & Do you have anxiety? & $138(71.5)$ & $55(28.5)$ \\
\hline 12. & Are you a smoker? & $15(7.8)$ & $178(92.2)$ \\
\hline 13. & Do you have family history of irritable bowel syndrome? & $98(50.8)$ & $95(49.2)$ \\
\hline 14. & Do you exercise regularly? & $27(14.0)$ & $166(86.0)$ \\
\hline
\end{tabular}

\section{Discussion}

As nurses play a crucial role in all health settings [32-38], the present study was conduct to assess the prevalence of IBS among nursing students at king Abdul-Aziz University. The prevalence of irritable bowel syndrome various greatly among different studies, the present study estimated the prevalence of irritable bowel syndrome among the included study sample presented by 192 nursing students. Results of our study showed that the prevalence of irritable bowel syndrome using Rome VI criteria among undergraduate students at nursing faculty in King Abdul-Aziz University Jeddah, Saudi Arabia was $17.6 \%$.

If we now move to the international studies, a study that was conducted in Japan by Okami et al (2011) collected their data from several of nursing schools and medical schools located in Kyoto prefecture [28]. The prevalence in this study was $41.5 \%$ from predominant types were IBS-C with females which higher than those reported in other studies [28]. Another study that was done by Yang Liu et al (2014) which reveals that the prevalence of irritable bowel syndrome is $36.1 \%$ which support the preparation of medical students in Beijing, China have an even IBS and this is due to [24].

Regarding national studies, a study that was conducted by Alaqeel et al (2017) among medical students at King Saud bin Abdul-Aziz University of Health Sciences in Riyadh, reveals that the higher prevalence of IBS was more in female students at percentage of $25.9 \%$ [2]. On the other hand, a study was performed by Ibrahim et al (2013) among interns and medical students at King Abdul-Aziz University reported a prevalence of 51.5\% which is much higher than that recorded in our research [12]. Moreover, A study conducted among one thousand and three hundred fifty students in King Saud University in Riyadh done by Alturki et al. (2011) revealed a prevalence for IBS of $54.6 \%$ for females using Rome III criteria to diagnose IBS [26]

In the previous international and national studies, they are much higher than the prevalence that recorded in our research. And this is due to unhealthy food and psychological factors especially increase level of stressor among medical students, fluctuation of hormone levels in females, unhealthy habits, and low grade and sleep disorder give rise. The contradiction between the other studies and the current study may be due to the variation between the countries. As well as, due to dietary and cultural habits in difference countries, so the interest in the future goes to attempt to correlate between these differences. Other reason, the incidence rate of IBS is relatively different, the difference may be due to the design study, sampling selection, eligibility criteria which is characteristics that define the target population (inclusion criteria and exclusion criteria), factors influencing the diagnosis of IBS and diagnostic criteria used.

Other study was approximately equal to the prevalence of IBS that we determined. The reason for this increase is low quality of life in female gender and psychological factors especially stressful routine between medical students which leads to high prevalence of IBS In them, which is due to the study design, sampling selection, and diagnostic criteria used. A study done by Iftikhar et al (2018) Among five hundred and fifty-two students, "Students were selected from Khyber Medical College, Khyber Girls Medical College, Rehman Medical College, Peshawar Medical College and using simple random sampling technique in Peshawar at Pakistan. The prevalence for females was $17.5 \%$, ROME III criteria was used to determine IBS [27].

A lower prevalence $14.7 \%$ for females than that for our current study was reported from study conducted among nurses staff at King Abdul-Aziz University Hospital in Jeddah by Ibrahim et al (2016). Rome III Criteria was used to determine nurses with positive diagnosis of IBS [23]. This discrepancy between two studies may be due to the previous study was conducted among nursing working in the hospital that can face many challenges and conflicting such as being short staffed, long shifts, salary, discrimination and job hazards, or due to the time conducted of the two studies and diagnostic criteria were used.

Regarding to IBS risk factors, some factors are strongly associated with IBS such as psychological stress, insomnia, depression and anxiety. In our study reveals 138 students with a percentage of $71.5 \%$ are experiencing anxiety. In the psychological stress section of literature review study; A study of Alturki et al (2011) involving King Saud University students, psychological stress and insomnia were encountered more frequently in participants with IBS [26]. Other study conducted from 
Pakistan illustrates a relationship between anxiety and IBS among medical students, Out of 102 patients, 57 of total individuals were found diagnosed with anxiety. Therefore, almost $55.8 \%$ patients were found associated to anxiety [27]. The significant difference between these two studies and current study may be due to factors influencing the diagnosis of irritable bowel syndrome or diagnostic criteria used.

\section{Conclusion}

The study illustrated a prevalence of (17.6\%) of Petulant Bowel Condition between nursing students at King Abdul-Aziz University in Jeddah. The percentage of female students unmarried was high. The majority of the female students were between nineteenth and twenty-one. Our study was intended educational level headed for second-year nursing students, third and fourth year students. Some students noticed a change in their bowel habit. A high rate of recurrent abdominal pain also was reported. Students Show that they are experiencing anxiety, and higher percentage of them have family history of irritable bowel syndrome. In addition our result demonstrated that the majority of the nursing students do not exercise regularly, also our result reveals that there has been an impact of irritable bowel syndrome on students' academic performance.

\section{Recommendations}

According to these result, we recommend, regular screening for irritable bowel syndrome and psychological problems especially stress and anxiety by ministry of health among primary health care. Anxiety management options are required to support students to manage with unalike stressors through their medical educations and work. Because students who are frequently suffering from stress and anxiety are more exposed to irritable bowel syndrome, so regarding to that we recommend to ministry of education to provide relaxation room for students at colleges and also provide activity to reduce the stress.

Furthermore, The discoveries of the recent study point to the requirement for making programs talking the significant health apprehensions for nurses, specifically irritable bowel syndrome, food sensitivity also working conditions as it is play a major role in increasing stress. There residues a requirement for research that attentions on the medical filed also utilizes correct methods to improved determine the habits describe their symptoms then to recognize the influences that encourage people to access or self-treat, That affects the provision of care to the patients, because when the nurses are tired and stressed they will not be able to pay attention to the patients. So, it is necessary to consider this.

On the other hand, there is a need to develop a program that screens the symptoms of irritable bowel syndrome in the work and school's environment. Keeping in view the high occurrence of irritable bowel syndrome among medical colleges and university students, there is want to aware the schoolgirls from the potential negative results of this disorder. Exercise and its role in symptoms of irritable bowel syndrome. Chronic diseases and their effect in exacerbation of irritable bowel syndrome.

There are an amount of restrictions in our existing study. Initially, our data is created on a nominated collection of nursing students and fixes not mean the broad populace. Next, due to extreme capacity on nursing students and the added stress on students obligating their exam may ensure overstated their digestive indicators and emotional stressor the stipulations of surveys might not need been exactly full and it might effects the results. Thirdly, approximately studies that were accompanied between health students were not available. Furthermore, some readings conducted among medical students were based on convenience samples.

\section{Acknowledgements}

We would like to show our gratitude to Dr.Fatmah Alshareef assistant professor in medical surgical nursing at King Abdulaziz University, nursing college. she was our mentor and she gave us a big support during this research project. We also would like to thank our college of nursing for giving us the opportunity to work on such a research. In addition, we also would like to thank Dr.Adnan Almezani who shared his questionnaire with us. Finally, we would like to thank the participants who played great role in facilitating our research.

\section{References}

[1] Jeffery, I. B., O'Toole, P. W., Öhman, L., Claesson, M. J., Deane, J., Quigley, E. M., \& Simrén, M. (2012, July 01). An irritable bowel syndrome subtype defined by species-specific alterations in faecal microbiota. Retrieved from https://gut.bmj.com/content/61/7/997.

[2] Alaqeel, M. K., Alowaimer, N. A., Alonezan, A. F., Almegbel, N. Y., \& Alaujan, F. Y. (2017). Prevalence of irritable bowel syndrome and its association with anxiety among medical students at King Saud bin Abdulaziz University for Health Sciences in Riyadh. Pakistan journal of medical sciences, 33(1), 33.

[3] Barbara, G., De Giorgio, R., Stanghellini, V., Cremon, C., Salvioli, B., \& Corinaldesi, R. (2019). New pathophysiological mechanisms in irritable bowel syndrome.

[4] Walker, B. R., Colledge, N. R., Penman, I. D., \& Ralston, S. H. (2014). Davidsons principles and practice of medicine.

[5] Danish, M. I. (2012). Short textbook of medical diagnosis and management. New Delhi: Med Tech.

[6] Brunner, L. S., Suddarth, D. S., Smeltzer, S. C., \& Bare, B. G. (2014). Brunner \& Suddarths textbook of medical-surgical nursing. Philadelphia: Lippincott Williams \& Wilkins

[7] Weaver, K., Melkus, G. and Henderson, W. (2017). Irritable Bowel Syndrome. [online] American Journal of Nursing. Available at:

https://journals.lww.com/ajnonline/Fulltext/2017/06000/Irritable_ Bowel_Syndrome.26.aspx [Accessed 28 Jan. 2019].

[8] Waehrens, R. (2018). Epidemiology of Irritable Bowel Syndrome hereditary and non-hereditary factors.)

[9] Enck, P., Aziz, Q., Barbara, G., Farmer, A., Fukudo, S., \& Mayer, E. et al. (2019). Irritable bowel syndrome.

[10] Chang, L. (2019). Updates to the Rome Criteria for Irritable Bowel Syndrome. Retrieved from https:/www.ncbi.nlm.nih.gov/pmc/articles/PMC5479345/.

[11] Mostafa, R. (2019). Rome III: The functional gastrointestinal disorders, third edition, 2006.

[12] Ibrahim, N. K. R., Battarjee, W. F., \& Almehmadi, S. A. (2013). Prevalence and predictors of irritable bowel syndrome among medical students and interns in King Abdulaziz University, Jeddah. The Libyan Journal Of Medicine, 8(1), 21287-21287. 
[13] Schmulson, M. and Drossman, D. (2019). What Is New in Rome IV.

[14] Hovdenak, N. (1987). Loperamide Treatment of the Irritable Bowel Syndrome. Scandinavian Journal Of Gastroenterology, 22(sup130), 81-84.

[15] Mostafa H., Yousef F., Hassan H. Health Related Quality of Life Educational Interventions: Effect on Chronic Hepatitis C Patients'. Saudi Journal of Nursing and Health Care. 2018; 1(2): 56-67.

[16] Hassan H. Effectiveness of a structured teaching program on anxiety and perception regarding toxoplasmosis among seropositive pregnant women in Northern Upper Egypt. Clinical Nursing Studies. 2018; 6(1): 1-19.

[17] Hassanine Sh., Hassan H., Alkotb Z. Effect of Preventive Program on Progression of Osteoporosis among Female Patients over 40 years at El-Fayoum City. American Research Journal of Nursing, 2017; 3(1): 1-15.

[18] Hassan H. The Impact of Evidence-Based Nursing as the Foundation for Professional Maternity Nursing Practices. Open Access Journal of Reproductive system and Sexual Disorder, 2019; 2(2): 196-197.

[19] Emam E., Abd El Rheem A., Ghanem N., Hassan H. Knowledge and Attitude of Women and Nurses regarding Pre-Conception Care: A Comparative Study. American Research Journal of Nursing, 2019; 5(1): 1-15.

[20] Farag D. \& Hassan H. Maternal Postpartum Sleep disturbance and Fatigue: Factors Influencing. ARC Journal of Nursing and Healthcare, 2-19; 5(2): 27-40.

[21] Atwa A., Hassan H., Ahmed S. The impact of a hospital-based awareness program on the knowledge of patients about breas cancer and cancer cervix. International Journal of Studies in Nursing. 2019; 4(1): 20-29.

[22] Irvine, E. J. (2004). Tegaserod prompts global relief of symptoms in the irritable bowel syndrome. ACP journal club, 141(2), 44-44.

[23] Ibrahim, N. K., Al-Bloushy, R. I., Sait, S. H., Al-Azhary, H. W., Bar, N. H. A., \& Mirdad, G. A. (2016). Irritable bowel syndrome among nurses working in King Abdulaziz University Hospital, Jeddah, Saudi Arabia. The Libyan Journal Of Medicine, 11(1), 30866-30866.

[24] Liu, Y., Liu, L., Yang, Y., He, Y., Zhang, Y., Wang, M., \& Yao, S. (2014). A school-based study of irritable bowel syndrome in medical students in beijing, china: prevalence and some related factors. Gastroenterology research and practice, 2014.

[25] Saad Naeem, S., Umar Siddiqui, E., Nafey Kazi, A., Amin Memon, A., Tauseeq Khan, S., \& Ahmed, B. (2012). Prevalence and factors associated with irritable bowel syndrome among medical students of Karachi, Pakistan: A cross-sectional study. BMC Research Notes, 5(1), 255-259.

[26] Al-Turki, Y. A., Aljulii, M. Z., Al Murayshid, A., Al Omaish, H. R., Al Daghiri, K. S., Al Seleemi, A. Y., . . . Al Jerawi, A. I. (2011). Prevalence of Irritable Bowel Syndrome among Students in King Saud University, Riyadh, Saudi Arabia. Middle East Journal of Family Medicine, 9(5), 17-20.

[27] Hassan H. Integrative Nursing Science in Women's Preconceptional Wellness. International Journal of Health and Biological Sciences, 2019; 2(1): 17-18.

[28] Okami, Y., Kato, T., Nin, G., Harada, K., Aoi, W., Wada, S., ... \& Kanazawa, M. (2011). Lifestyle and psychological factors related to irritable bowel syndrome in nursing and medical school students. Journal of gastroenterology, 46(12), 1403-1410.'

[29] Terry, A. (2012). Clinical research for the doctor of nursing practice.

[30] Bluman, A. (2015). Elementary Statistics: a Step by Step Approach. London: McGraw-Hill Education - Europe.

[31] Almezani, A. M. M., Alkhalaf, A. A., Alharbi, M. N. K., Alhuwayfi, S. A., Alshammari, H. M., Alshammari, M. S., ... \& Alqasem, A. A. (2018). Prevalence of Irritable Bowel Syndrome among Medical Students in Hail University, Saudi Arabia. The Egyptian Journal of Hospital Medicine, 71(2), 2581-2585.

[32] Hassan H. Call for psychosocial well-being among pregnant women associated with medical disorder in Beni-Suef governorate. IOSR Journal of Nursing and Health Science. 2016; 5(2): 81-94.

[33] Sheha E., Hassan H., Gamel W. Association between pre-pregnant overweight and obesity and periodontal disease during pregnancy: a cross sectional study. International Journal of Studies in Nursing. 2018; 3(1): 1-21.

[34] Hassan H. Infertility profile, psychological ramifications and reproductive tract infection among infertile women, in northern Upper Egypt. Journal of Nursing Education and Practice. 2016; 6(4): 92-108.

[35] Faheim S., Hassan H., Gamel W. Topical Application of Human Milk versus Alcohol and Povidine-Iodine on Clinical Outcomes of Umbilical Cord in Healthy Newborn: Impact of an Educational Program of Mothers. International Journal of Studies in Nursing, 2019; 4(2): 35-51.

[36] Hassan H, Nasr E. Improving nurses' knowledge and skills regarding tocolytics for inhibiting preterm labor. Clinical Nursing Studies. 2017; 5(1): 1-12.

[37] Farg D. and Hassan H. Study Hyperemesis Graviderum Requiring Hospital Admission during Pregnancy: Effect of Nursing Implication on Its Progress. American Journal of Nursing Research, 2019; 7(2): 328-341.

[38] Hassan H \& Farag D. The impact of polycystic ovary syndrome on women's quality of life: Nursing guidelines for its management Clinical Nursing Studies, 2019, 7(3): 42-57.

[39] Alsuwailm, W. A., AL-Qahtani, M. M., AL-Hulaibi, A. A., ALHadi, M. A., Ali, S. I., \& Shehabeldeen, S. A. (2017). Irritable Bowel Syndrome among Medical Students and Interns in King Faisal University. Open Journal of Preventive Medicine, 7(11), 235

[40] Lacy, B. and Patel, N. (2019). Rome Criteria and a Diagnostic Approach to Irritable Bowel Syndrome.

(C) The Author(s) 2019. This article is an open access article distributed under the terms and conditions of the Creative Commons Attribution (CC BY) license (http://creativecommons.org/licenses/by/4.0/). 